\title{
ON THE ELECTROSTATIC POTENTIAL DUE TO A SLAB SHAPED AND A SEMI-INFINITE NaCl-TYPE LATTICE
}

\author{
B.R.A. NIJBOER \\ Institute of Theoretical Physics, University of Utrecht, Princetonplein 5, Utrecht, The Netherlands
}

\author{
Received 29 August 1983
}

\begin{abstract}
Consider $N$ layers of a NaCl-type ionic lattice such that in every layer one has an infinite square lattice of positive and negative unit point charges. We present formulae in which the electrostatic potential in an arbitrary field point is expressed as a sum of two rapidly converging lattice sums. For $N \rightarrow \infty$ we obtain formulae applicable for a semi-infinite lattice.
\end{abstract}

\section{Introduction}

The problem of calculating the electrostatic potential in an arbitrary field point in an infinite lattice of point charges with a neutral basis has been treated first in a well-known paper by $P$. Ewald ${ }^{1}$ ). By an ingenious method Ewald converts the slowly convergent lattice sum representing the potential into two rapidly converging lattice sums. Since then his method has been varied, extended and applied by a great many authors. A recent review of the relevant literature has been published by Glasser and Zucker ${ }^{2}$ ). Of late several authors have again drawn attention to the fact that the original lattice sum is only conditionally convergent, so that its value depends on the order of summation or expressed in a different way, that for a large crystal the result for the potential may be shape dependent.

E.R. Smith in a very recent paper in this journal ${ }^{3}$ ) which is mainly devoted to the calculation of the electrostatic potential at a plane surface of a slab shaped piece of a lattice of point ions, also rederives Ewald's result for an "infinite" lattice, including some terms which in a general case will be dependent on its shape. Here I would like to point out that twenty five years ago de Wette and I have calculated the electrostatic potential in an infinite lattice of point charges with a neutral basis in a way different from that of Ewald ${ }^{4}$ ). Again a slowly convergent lattice sum is expressed as the sum of rapidly converging lattice sums, but whereas according to Ewald's method these sums have to be calculated anew for every field point, in our method the final rapidly converging sums do not depend on the field point. They can be calculated once for all for a given lattice. Some numerical results are also reproduced. In ref. 4 (and earlier papers) we gave. 
a rather extensive discussion of a possible shape dependence as a result of the conditional convergence of the original lattice sum. In particular it was shown that if the neutral basis has no dipole moment (which is the usual case), but does have a quadrupole moment, the potential has a shape dependent contribution. This contribution is, however, independent of position, so that the electric field is not affected. This conclusion has been confirmed by Smith $^{3}$ ).

In the present paper I propose to apply the old methods of de Wette and myself ${ }^{5}$ ) to a problem similar to the main subject to Smith's ${ }^{3}$ ) paper, namely to calculate the electrostatic potential in an arbitrary field point due to a slab of an ionic lattice with a neutral basis and also that due to a semi-infinite ionic lattice. I will restrict myself to the simple case of a $\mathrm{NaCl}$-type lattice. Smith treats a more general case, but it will be seen that his results are also more complicated than those presented here.

\section{Potential due to a slab of parallel planes of a $\mathrm{NaCl}$-type lattice}

Let us consider a square lattice (lattice constant 1), situated in the $x y$-plane, the lattice points of which are alternatively occupied by positive and negative unit charges. We choose the origin of coordinates in a lattice point with positive unit charge.

The electrostatic potential in an arbitrary field point $x, y, z$ (where to begin with we assume that $0<x<1$ and $0<y<1$ ) is given by

$$
\phi(x, y, z)=\sum_{n_{1}=-\infty}^{+\infty} \sum_{n_{2}=-\infty}^{+\infty}(-1)^{n_{1}+n_{2}}\left\{\left(n_{1}-x\right)^{2}+\left(n_{2}-y\right)^{2}+z^{2}\right\}^{-1 / 2} .
$$

If we introduce the 2-dimensional vectors $\boldsymbol{R}(x, y)$ and $n\left(n_{1}, n_{2}\right)$ we may write

$$
\phi(x, y, z)=\int \sum_{n_{1}=-\infty}^{+\infty} \sum_{n_{2}=-\infty}^{+\infty}(-1)^{n_{1}+n_{2}} \delta(\boldsymbol{r}-\boldsymbol{n})\left\{(\boldsymbol{r}-\boldsymbol{R})^{2}+z^{2}\right\}^{-1 / 2} \mathrm{~d}^{2} r .
$$

Taking 2-dimensional Fourier transforms:

$$
\begin{aligned}
\int \mathrm{e}^{2 \pi \mathrm{i} h \cdot r} \sum_{n_{1}} \sum_{n_{2}}(-1)^{n_{1}+n_{2}} \delta(\boldsymbol{r}-\boldsymbol{n}) \mathrm{d}^{2} r & =\sum_{n_{1}} \sum_{n_{2}} \mathrm{e}^{\mathrm{i} \pi\left(n_{1}+n_{2}\right)} \mathrm{e}^{2 \pi i h \cdot n} \\
& =\sum_{m_{1}=-\infty}^{+\infty} \sum_{m_{2}=-\infty}^{+\infty} \delta\left(\boldsymbol{h}-\left(\boldsymbol{m}+\frac{1}{2}\right)\right), \\
\int \mathrm{e}^{2 \pi i h \cdot r}\left\{(\boldsymbol{r}-\boldsymbol{R})^{2}+z^{2}\right\}-1 / 2 \mathrm{~d}^{2} r & =\mathrm{e}^{2 \pi i h \cdot R} \int \mathrm{e}^{2 \pi i h \cdot r}\left(r^{\prime 2}+z^{2}\right)^{-1 / 2} \mathrm{~d}^{2} r^{\prime} \\
= & \mathrm{e}^{2 \pi i h \cdot R} h^{-1} \mathrm{e}^{-2 \pi h|z|},
\end{aligned}
$$


we obtain, applying Parseval's theorem,

$$
\begin{aligned}
\phi(x, y, z)= & \int \sum_{m_{1}} \sum_{m_{2}} \delta\left(\boldsymbol{h}-\left(\boldsymbol{m}+\frac{1}{2}\right)\right) \mathrm{e}^{2 \pi \mathrm{i} h \cdot \boldsymbol{R}} \mathrm{e}^{-2 \pi h \mid-1} h^{-1} \mathrm{~d}^{2} h \\
= & \sum_{m_{1}=-\infty}^{\infty} \sum_{m_{2}=-\infty}^{+\infty}\left\{\left(m_{1}+\frac{1}{2}\right)^{2}+\left(m_{2}+\frac{1}{2}\right)^{2}\right\}^{-1 / 2} \\
& \times \exp \left\{-2 \pi|z|\left(\left(m_{1}+\frac{1}{2}\right)^{2}+\left(m_{2}+\frac{1}{2}\right)^{2}\right)\right\}^{1 / 2} \exp \left\{2 \pi \mathrm{i} \boldsymbol{R} \cdot\left(\boldsymbol{m}+\frac{1}{2}\right)\right\} .
\end{aligned}
$$

For $x=y=z=0$ this expression diverges. Let us therefore consider first the case $z>0$.

Now take a slab of $N$ parallel planes at $z=0, z=-1, \ldots, z=-(N-1)$, in such a way that alternately positive and negative point charges are situated immediately below each other.

As

$$
\sum_{n=0}^{N-1}(-1)^{n} \mathrm{e}^{-2 \pi n \alpha}=\frac{1-(-1)^{N} \mathrm{e}^{-2 \pi N \alpha}}{1+\mathrm{e}^{-2 \pi \alpha}},
$$

the potential at $x, y, z>0$ due to this slab is found to be

$$
\phi_{N}(x, y, z)=\sum_{m_{1}=-\infty}^{+\infty} \sum_{m_{2}=-\infty}^{+\infty} \alpha_{m}^{-1} \mathrm{e}^{-2 \pi z \alpha_{m}}\left(\frac{1-(-1)^{N} \mathrm{e}^{-2 \pi N \alpha_{m}}}{1+\mathrm{e}^{-2 \pi x_{m}}}\right) \mathrm{e}^{2 \pi i R \cdot\left(m+\frac{1}{2}\right)},
$$

where we have put $\alpha_{m}=\left\{\left(m_{1}+\frac{1}{2}\right)^{2}+\left(m_{2}+\frac{1}{2}\right)^{2}\right\}^{1 / 2}$.

\section{Potential of a semi-infinite NaCl-type lattice}

If we let $N \rightarrow \infty$ we obtain the potential at $x, y, z>0$ of a semi-infinite $\mathrm{NaCl}$-type lattice of point charges:

$$
\phi_{\infty}(x, y, z)=\sum_{m_{1}=-\infty}^{+\infty} \sum_{m_{2}=-\infty}^{+\infty} \alpha_{m}^{-1} \mathrm{e}^{-2 \pi z x_{m}}\left(1+\mathrm{e}^{-2 \pi x_{m}}\right)^{-1} \mathrm{e}^{2 \pi \mathrm{iR} \cdot\left(m+\frac{1}{2}\right)},
$$

which may be written also

$$
\begin{aligned}
\phi_{\infty}(x, y, z)= & \sum_{m_{1}=0}^{\infty} \sum_{m_{2}=0}^{\infty} 4\left\{\alpha_{m}\left(1+\mathrm{e}^{-2 \pi \alpha_{m}}\right)\right\}^{-1} \\
& \times \mathrm{e}^{-2 \pi z z_{m}} \cos 2 \pi\left(m_{1}+\frac{1}{2}\right) x \cos 2 \pi\left(m_{2}+\frac{1}{2}\right) y .
\end{aligned}
$$

One easily verifies that $\phi(x+1, y, z)=\phi(x, y+1, z)=-\phi(x, y, z)$, as it should. Unless $z$ is very small the series (8) converges rapidly. If $z$ is very small, one can make a Taylor-expansion in powers of $z^{2}$ of (1) in the contribution of the plane $z=0$ and treat the successive terms by a method analogous to that described below. 
For $z=0$ it is preferable (and if in addition $x=y=0$ it is necessary) to evaluate the contribution of the plane $z=0$ separately. The contribution of the planes $z=-1, z=-2, \ldots$ amounts to

$$
\sum_{m_{1}=0}^{\infty} \sum_{m_{2}=0}^{\infty}-4\left\{\alpha_{m}\left(\mathrm{e}^{2 \pi a_{m}}+1\right)\right\}^{-1} \cos 2 \pi\left(m_{1}+\frac{1}{2}\right) x \cos 2 \pi\left(m_{2}+\frac{1}{2}\right) y .
$$

Now the contribution of the plane $z=0$. Let us first assume that $x$ and $y$ are not both 0 . We must calculate

$$
\sum_{n_{1}=-x_{1} n_{2}=-\infty}^{+\infty} \sum^{+\infty}(-1)^{n_{1}+n_{2}}\left\{\left(n_{1}-x\right)^{2}+\left(n_{2}-y\right)^{2}\right\}^{-1 / 2} \text {. }
$$

This sum will be treated according to the method explained in detail in ref. 5 . (10) can be written as

$$
\begin{aligned}
\int_{n_{1}=-\infty}^{+\infty} \sum_{n_{2}=-\infty}^{+\infty}(-1)^{n_{1}+n_{2}} \delta(\boldsymbol{r}-\boldsymbol{n}) \frac{1}{|\boldsymbol{r}-\boldsymbol{R}|} \mathrm{d}^{2} r \\
=\int \sum_{n_{1}=-\infty}^{+\infty} \sum_{n_{2}=-\infty}^{+\infty}(-1)^{n_{1}+n_{2}} \delta(\boldsymbol{r}-\boldsymbol{n}) \frac{\phi(\sqrt{\pi}|\boldsymbol{r}-\boldsymbol{R}|)}{|\boldsymbol{r}-\boldsymbol{R}|} \mathrm{d}^{2} \boldsymbol{r} \\
\quad+\int \sum_{n_{1}=-\infty}^{+\infty} \sum_{n_{2}=-\infty}^{+\infty}(-)^{n_{1}+n_{2}} \delta(\boldsymbol{r}-\boldsymbol{n}) \frac{1-\phi(\sqrt{\pi}|\boldsymbol{r}-\boldsymbol{R}|)}{|\boldsymbol{r}-\boldsymbol{R}|} \mathrm{d}^{2} r .
\end{aligned}
$$

Here

$$
\phi(x)=\frac{2}{\sqrt{\pi}} \int_{x}^{\infty} \mathrm{e}^{-t^{2}} \mathrm{~d} t=\operatorname{erf}(x) .
$$

Since

$$
\int \frac{1-\phi(\sqrt{\pi} r)}{r} \mathrm{e}^{2 \pi i h \cdot r} \mathrm{~d}^{2} r=\frac{\phi(\sqrt{\pi} h)}{h} \quad \text { (2-dimensional Fourier transform), }
$$

we can write the second integral by Parseval's theorem as

$$
\int_{m_{1}=-\infty}^{+\infty} \sum_{m_{2}=-\infty}^{+\infty} \frac{\phi(\sqrt{\pi} h)}{h} \delta\left(\boldsymbol{h}-\left(\boldsymbol{m}+\frac{1}{2}\right)\right)^{2 \pi i R \cdot h} \mathrm{~d}^{2} h .
$$

We then find for the sum (10) the following expression:

$$
\begin{aligned}
& \sum_{n_{1}=-\infty}^{+\infty} \sum_{n_{2}=-\infty}^{+\infty}(-1)^{n_{1}+n_{2}} \frac{\phi(\sqrt{\pi}|\boldsymbol{n}-\boldsymbol{R}|)}{|\boldsymbol{n}-\boldsymbol{R}|}+\sum_{m_{1}=-\infty}^{+\infty} \sum_{m_{2}=-\infty}^{+\infty} \frac{\phi\left(\sqrt{\pi} \alpha_{m}\right)}{\alpha_{m}} \mathrm{e}^{2 \pi i \boldsymbol{R} \cdot\left(\boldsymbol{m}+\frac{1}{2}\right)} \\
& =\sum_{n_{1}=-\infty}^{+\infty} \sum_{n_{2}=-\infty}^{+\infty}(-1)^{n_{1}+n_{2}} \frac{\phi(\sqrt{\pi}|\boldsymbol{n}-\boldsymbol{R}|)}{|\boldsymbol{n}-\boldsymbol{R}|} \\
& \left.\quad+\sum_{m_{1}=0}^{\infty} \sum_{m_{2}=0}^{\infty} 4 \frac{\phi\left(\sqrt{\pi} \alpha_{m}\right)}{\alpha_{m}} \cos 2 \pi\left(m_{1}+\frac{1}{2}\right) x \cos 2 \pi\left(m_{2}+\frac{1}{2}\right) y\right) .
\end{aligned}
$$


Here again $\alpha_{m}=\left\{\left(m_{1}+\frac{1}{2}\right)^{2}+\left(m_{2}+\frac{1}{2}\right)^{2}\right\}^{1 / 2}$ and $|\boldsymbol{n}-\boldsymbol{R}|=\left\{\left(n_{1}-x\right)^{2}+\left(n_{2}-y\right)^{2}\right\}^{1 / 2}$. The total potential at $z=0$ due to the semi-infinite lattice is given by

$$
\phi(x, y, 0)=(9)+(11) .
$$

If $x=y=z=0$ we must subtract the contribution of the ion at the origin. Then the plane $z=0$ contributes

$$
\begin{aligned}
\sum_{n_{1} n_{2}}^{\prime}(-1)^{n_{1}+n_{2}}\left(n_{1}^{2}+n_{2}^{2}\right)^{-1 / 2}= & -2+\sum_{n_{1}=-\infty}^{+\infty} \sum_{n_{2}=-\infty}^{+\infty}(-1)^{n_{1}+n_{2}} \frac{\phi\left\{\sqrt{\pi\left(n_{1}^{2}+n_{2}^{2}\right)}\right\}}{\sqrt{n_{1}^{2}+n_{2}^{2}}} \\
& +\sum_{m_{1}=0}^{+\infty} \sum_{m_{2}=0}^{+\infty} 4 \frac{\phi\left(\sqrt{\pi} \alpha_{m}\right)}{\alpha_{m}} .
\end{aligned}
$$

The dash at the summation signs means that the term $n_{1}=0, n_{2}=0$ should be omitted. It will be evident that the above results may be generalized in a straightforward way to the case of more complicated ionic lattices. And further the case $z<0$ could be treated in a similar way. It should be noted that related work, also based on our method of planewise summation, has been published recently by V. Massidda ${ }^{6}$ ), where further references may be found.

\section{References}

1) P. Ewald, Ann. Phys. 64 (1921) 253.

Cf. also M. Born, and M. Göppert-Mayer, Hdb. Physik, vol. 24, part 2 (Springer, Berlin, 1933) p. 710 .

2) M.L. Glasser and I.J. Zucker, in: Theoretical Chemistry: Advances and Perspectives, vol. 5 (Academic Press, New York, 1980) p. 67.

3) E.R. Smith, Physica 120A (1983) 327; and other papers cited there.

4) F.W. de Wette and B.R.A. Nijboer, Physica 24 (1958) 1105 (cf. in particular pp. 1107 and 1111).

5) B.R.A. Nijboer and F.W. de Wette, Physica 23 (1957) 309; 24 (1958) 422. F.W. de Wette, thesis, Utrecht 1959.

6) V. Massidda, Physica 85 B (1976) 311; 95 B (1978) 317. 